\title{
An orbit fit program for localizing errors in RHIC
}

\author{
C. Liu, M.Minty, V. Ptitsyn
}

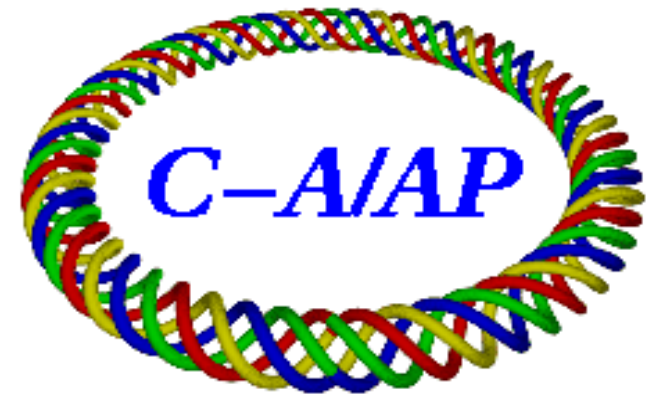

\section{Collider-Accelerator Department Brookhaven National Laboratory \\ Upton, NY 11973}

Notice: This document has been authorized by employees of Brookhaven Science Associates, LLCunder Contract No. DE-AC02-98CH10886 with the U.S. Departm ent of En ergy. The United States Governm ent retains a nonexclusive, paid-up, irrevocable, world-wide license topublish or reproduce the published formof this document, or allow others to do so, for United States Government purposes. 


\title{
An orbit fit program for localizing errors in RHIC
}

\author{
C. Liu* and M. Minty, V. Ptitsyn \\ BNL, Upton, NY, U.S.A.
}

(Dated: October 18, 2011)

\begin{abstract}
Many errors in an accelerator are evidenced as transverse kicks to the beam which distort the beam trajectory. Therefore, the information of the errors are imprinted in the distorted orbits, which are different from what would be predicted by the optics model. In this note, we introduce an algorithm for fitting the orbit based on an on-line optics model. By comparing the measured and fitted orbits, we first present results validating the algorithm. We then apply the algorithm and localize the location of the elusive source of vertical diurnal variations observed in RHIC.
\end{abstract}

\section{INTRODUCTION}

The difference of two trajectories (linear accelerator) or closed orbits (storage ring) should match exactly a betatron ocsillation, which is predictable by the optics model, in an ideal machine. However, in the presence of errors, the measured trajectory deviates from prediction since the model is imperfect. Comparison of measurement to model can be used to detect such errors. To do so the initial conditions (phase space parameters at any point) must be determined which can be done by comparing the difference orbit to prediction using only a few beam position monitors (BPMs). The fitted orbit can be propagated along the beam line based on the optics model. Measurement and model will agree up to the point of an error. The error source can be better localized by additionally fitting the difference orbit using downstream BPMs and back-propagating the solution. If one dominating error source exist in the machine, the fitted orbit will deviate from the difference orbit at the same point $[1,2]$.

\section{ALGORITHM}

In either a transport line or a storage ring, the beam trajectory in either transverse plane (neglecting coupling) can be expressed by the transfer matrix and initial conditions,

$$
z(s)=\sqrt{\frac{\beta_{s}}{\beta_{i}}}\left(\cos \left(\phi_{s}-\phi_{i}\right)+\alpha_{i} \sin \left(\phi_{s}-\phi_{i}\right)\right) \cdot z_{i}+\sqrt{\beta_{s} \beta_{i}} \sin \left(\phi_{s}-\phi_{i}\right) \cdot z_{i}^{\prime},
$$

where $\beta_{s}, \beta_{i}, \phi_{s}, \phi_{i}$ are the beta functions and phase advances at position $s$ and initial position $i$; $\alpha_{i}$ is the initial alpha and $z$ represents either the horizontal $(x)$ or vertical $(y)$ motion. The following algorithm may be applied to both beam trajectory in linear machine and closed orbit in storage ring, which afterwards we will refer to as "orbit".

The difference of two orbits comply with

$$
\triangle z=M_{11} \cdot \triangle z_{i}+M_{12} \cdot \triangle z_{i}^{\prime}
$$

here $M_{11}=\sqrt{\frac{\beta_{s}}{\beta_{i}}}\left(\cos \left(\phi_{s}-\phi_{i}\right)+\alpha_{i} \sin \left(\phi_{s}-\phi_{i}\right)\right), M_{12}=\sqrt{\beta_{s} \beta_{i}} \sin \left(\phi_{s}-\phi_{i}\right)$.

By selecting a fitting range from the $m$ th BPM to the $n$th BPM, a set of linear equations follows,

$$
\left\{\begin{array}{l}
\triangle z_{m}=M_{11}^{m} \cdot \triangle z_{i}+M_{12}^{m} \cdot \triangle z_{i}^{\prime} \\
\triangle z_{m+1}=M_{11}^{m+1} \cdot \triangle z_{i}+M_{12}^{m+1} \cdot \triangle z_{i}^{\prime} \\
\vdots \\
\triangle z_{n}=M_{11}^{n} \cdot \triangle z_{i}+M_{12}^{n} \cdot \triangle z_{i}^{\prime}
\end{array}\right.
$$

Using a least-square fit (minimizing the merit function $\left.f=\sum_{k=m}^{n}\left(\triangle z_{k}-M_{11}^{k} \cdot \triangle z_{i}+M_{12}^{k} \cdot \triangle z_{i}^{\prime}\right)^{2}\right)$, we obtain the

*cliu1@bnl.gov 
initial conditions as

$$
\left\{\begin{array}{l}
\triangle z_{i}=\frac{\sum_{k=m}^{n} \triangle z_{k} M_{11}^{k} \cdot \sum_{k=m}^{n} M_{11}^{k} M_{12}^{k}-\sum_{k=m}^{n} \triangle z_{k} M_{12}^{k} \cdot \sum_{k=m}^{n}\left(M_{11}^{k}\right)^{2}}{\left(\sum_{k=m}^{n} M_{11}^{k} M_{12}^{k}\right)^{2}-\sum_{k=m}^{n}\left(M_{11}^{k}\right)^{2} \cdot \sum_{k=m}^{n}\left(M_{12}^{k}\right)^{2}} \\
\triangle z_{i}^{\prime}=\frac{\sum_{k=m}^{n} \triangle z_{k} M_{11}^{k} \cdot \sum_{k=m}^{n}\left(M_{12}^{k}\right)^{2}-\sum_{k=m}^{n} \triangle z_{k} M_{12}^{k} \cdot \sum_{k=m}^{n} M_{11}^{k} M_{12}^{k}}{\sum_{k=m}^{n}\left(M_{11}^{k}\right)^{2} \cdot \sum_{k=m}^{n}\left(M_{12}^{k}\right)^{2}-\left(\sum_{k=m}^{n} M_{11}^{k} M_{12}^{k}\right)^{2}}
\end{array}\right.
$$

Once the initial conditions are so determined, the fitted orbit along the ring can be computed based on Eqs. 1 .

\section{APPLICATION VERIFICATION}

This algorithm was integrated into RhicOrbitDisplay. As a demonstration, we use orbit data acquired during measurements made to check the BPM polarities. In RHIC, there is a possibility of BPM cable swaps following maintainence which would cause problems for orbit feedback which is now used for each acceleration cycle. The BPM polarity check works in this way. First the closed orbits before and after making a strength change of a corrector are recorded. The difference between those measurements, the so called difference orbit, is then formed. The difference is also predicted by the on-line model with knowledge of the corrector strength. A BPM polarity error can be identified when signs of the measured and predicted difference orbit do not agree.

As an after-fact test, we applied the algorithm and try to localize an error source, which in this case was due to the intentionally varied dipole corrector. The following horizontal orbit data was taken in the Blue ring, on Mar. 14th, 2011 (fill number: 15290). Shown in Fig. 1 are the measured horizontal difference orbit (blue) and the fitted

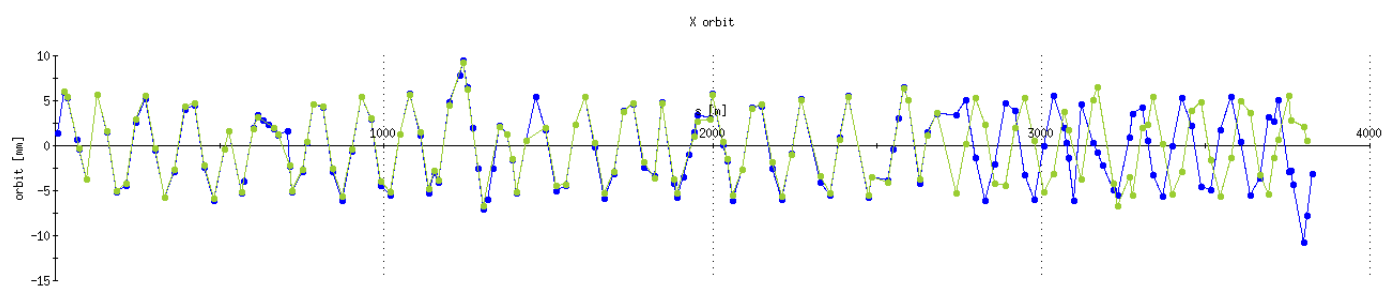

FIG. 1. Measured horizontal difference orbit (blue) versus the fitted orbit (cyan) by forwards propagation

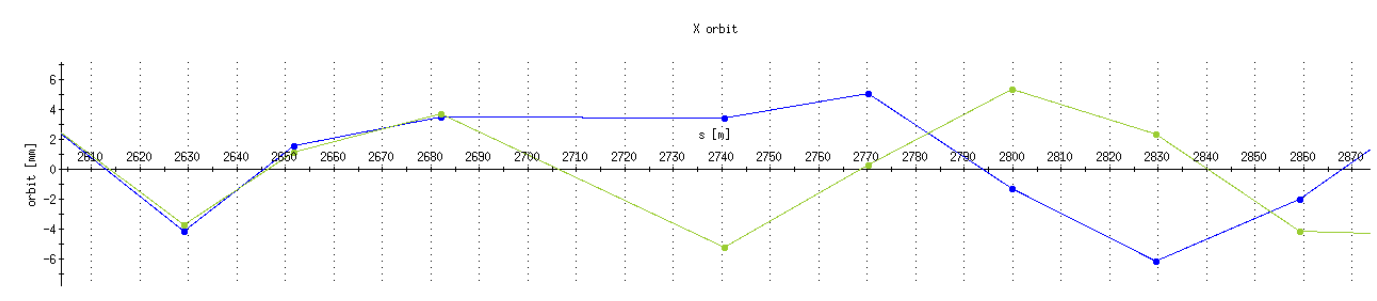

FIG. 2. The region (by zooming in Fig. 1) where the fitted orbit starts to deviate from the difference orbit

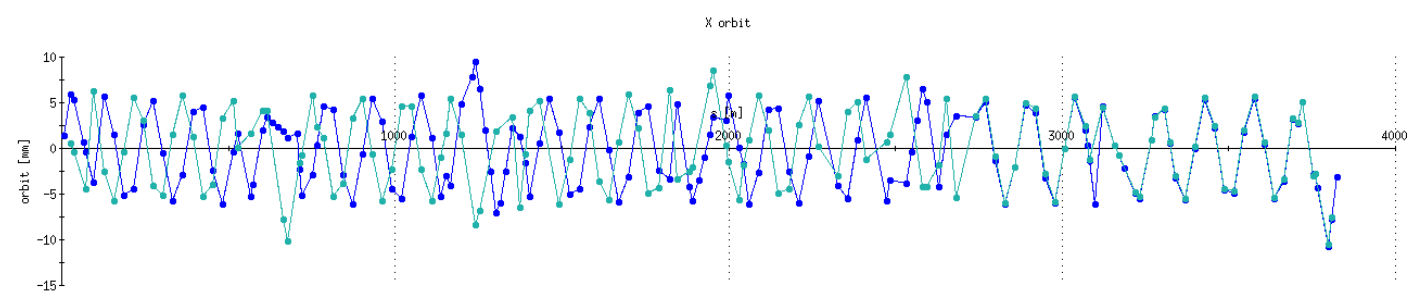

FIG. 3. Measured horizontal difference orbit (blue) versus the fitted orbit by backwards propagation 


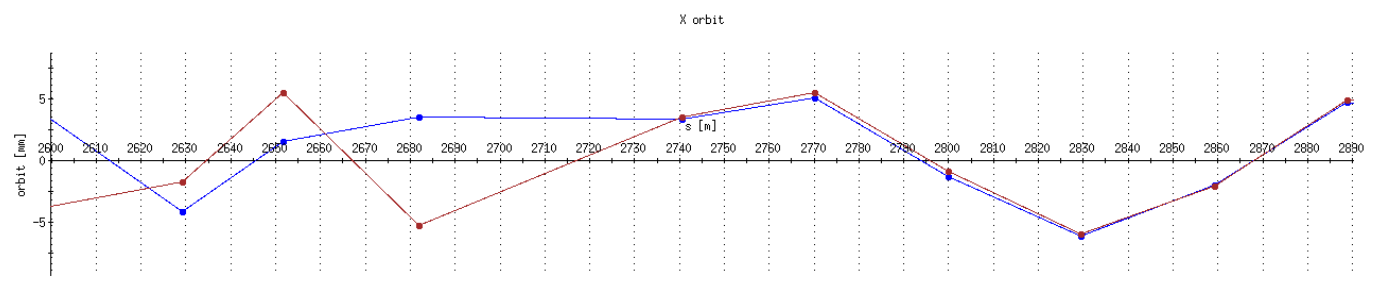

FIG. 4. The region (by zooming in Fig. 3) where the fitted orbit starts to deviate from the difference orbit

orbit (cyan). The fit range was 200 to $800 \mathrm{~m}$ and the fitted orbit was propagated forward. The agreement between measurement and fit is very good upstream of the BPM at $2686 \mathrm{~m}$. The expanded range shown in Fig. 2 reveals that the two orbits begin to deviate between BPMs located at 2683 and $2740 \mathrm{~m}$, which is in agreement with the longitudinal coordinate $(2713 \mathrm{~m}$ ) of the dipole magnet (bo2-th10) that was used. In order to confirm this result, a different region (3000 to $3600 \mathrm{~m}$ ) was selected for fitting and the resulting fitted orbit was propagated backwards for the same set of data. The result (Fig. 3) agrees with that in Fig. 1.

In the vertical plane with separation bumps, this algorithm works equally as well. The following vertical orbit data were taken in Yellow, on Jan. 17th, 2011 (fill number: 14822). Fig. 5 shows the fitted orbit (fitting range: 200 to

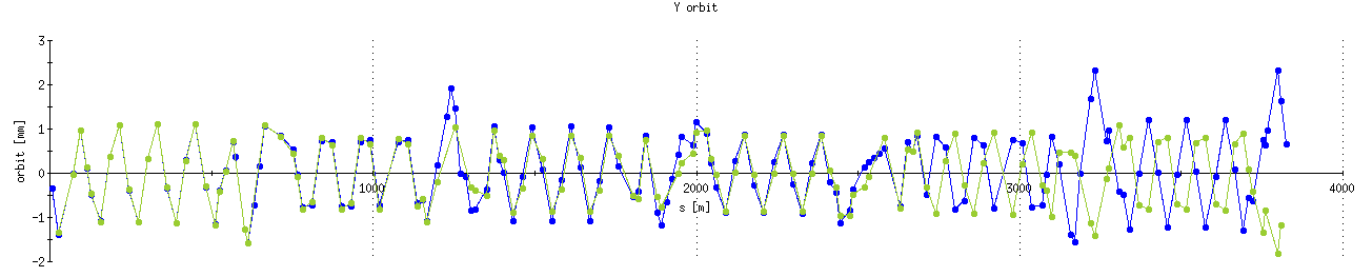

FIG. 5. Measured vertical difference orbit (blue) versus the fitted orbit by forwards propagation

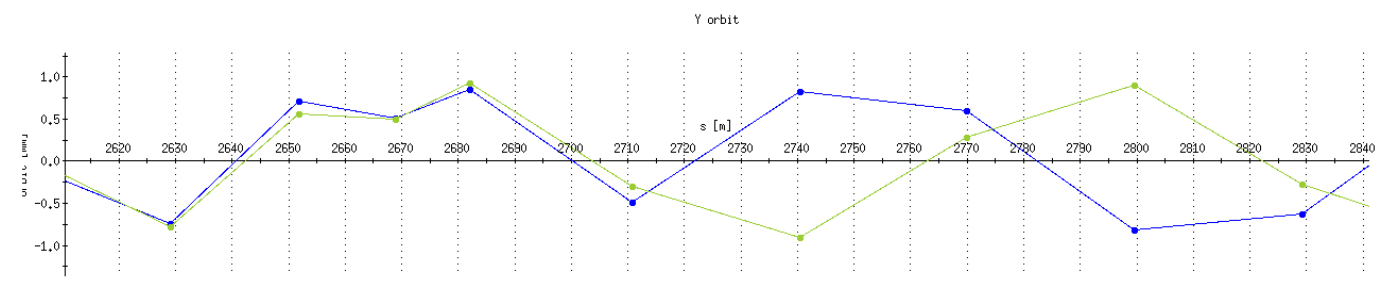

FIG. 6. The region (by zooming in Fig. 5) where the fitted orbit starts to deviate from the difference orbit

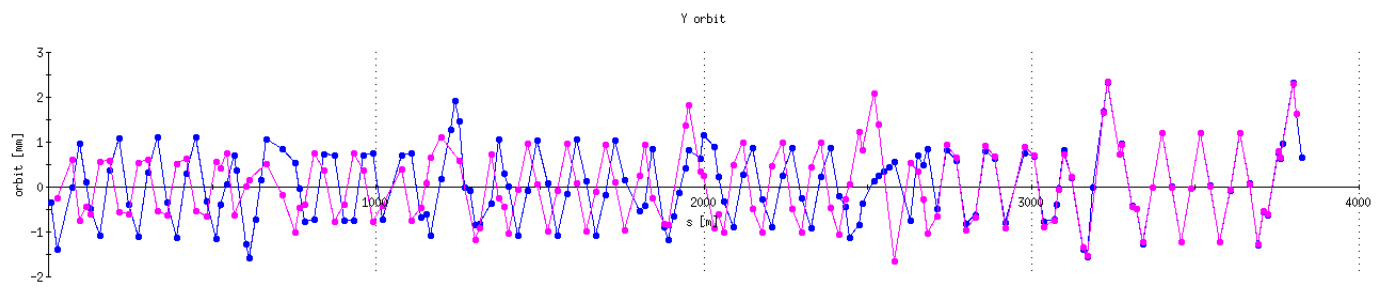

FIG. 7. Measured vertical difference orbit (blue) versus the fitted orbit by backwards propagation

$800 \mathrm{~m}$ ) versus measured difference orbit, which start to deviate from one another in between 2710 and $2740 \mathrm{~m}$ (in Fig. 6). The backwards propagated fitted orbit versus difference orbit (in Fig. 7) confirms the finding in Fig. 5. This agrees with the fact that the dipole magnet being used (yi2-tv10) was at $2713 \mathrm{~m}$.

We conclude from this analysis that the online orbit fit algorithm correctly localized an intentionally introduced perturbation. The localization was accurate with a range given by the distance between the two closest BPMs. 


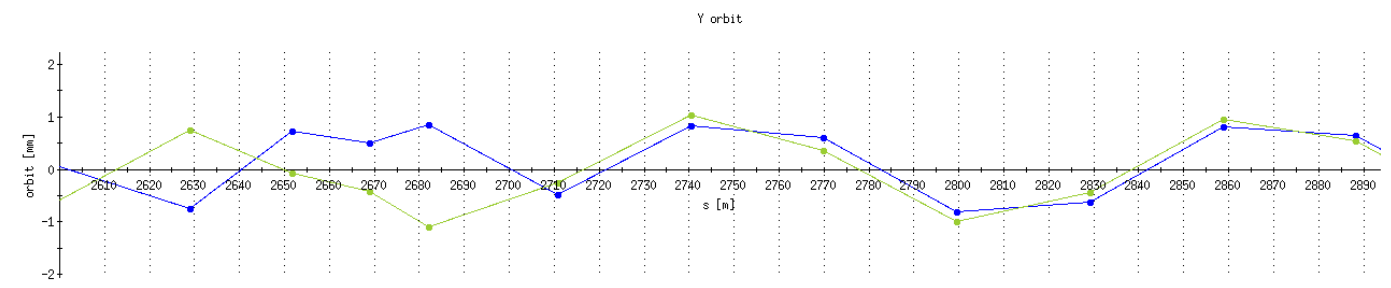

FIG. 8. The region (by zooming in Fig. 7) where the fitted orbit starts to deviate from the difference orbit

\section{LOCALIZATION OF DIURNAL DISTURBANCES IN RHIC}

Diurnal variations in the beam trajectory have long been observed at RHIC [3]. Using turn-by-turn BPM data [4], the source for vertical deviations was localized to near the IR at the vicinity of the accelerating cavities (IR4). The source was thought to originate near the cryogenic feed lines located there. As a test, these feed lines were mechanically decoupled from the roof of the accelerator tunnel [5], however, this did not seem to mitigate the diurnal perturbation to the beam trajectories.

To confirm this locale of diurnal variations, we applied the orbit fit algorithm to two sets of data. We took two orbit measurements at store (fillno: 15808, optics: Au11v1, time: 7:35:00 AM, 08:02:08 AM) and applied the orbit fit algorithm to the vertical difference orbit. First we used a range from 2000 to $2800 \mathrm{~m}$ for the fitting, and forward propagated the fitted orbit along the ring. The difference orbit and the fitted orbit were seen to deviate from one another near the 3 o'clock side of IR4. The details are clearer in Fig. 10 and Fig. 11 which shows the difference of the original difference orbit and the fitted orbit.

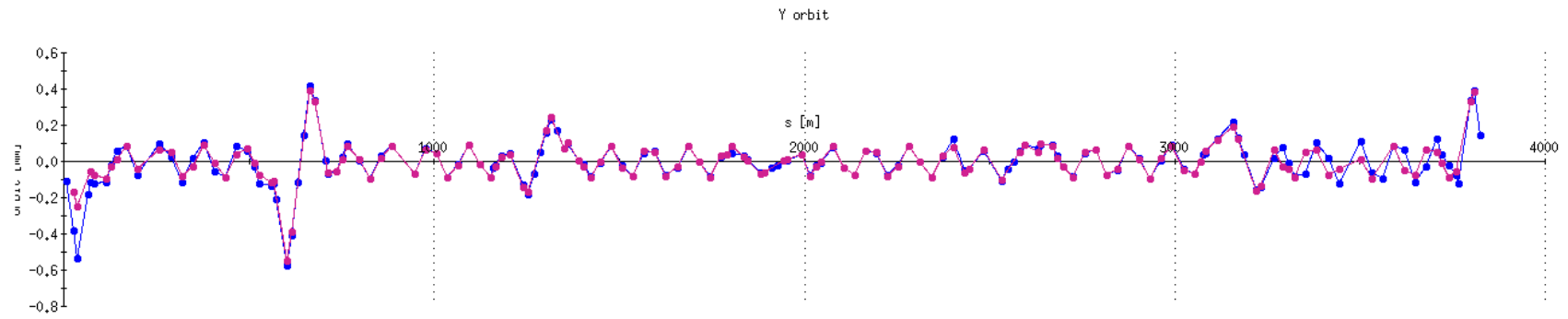

FIG. 9. The vertical difference orbit (blue) vs fitted orbit (red) using range from 2000 to $2800 \mathrm{~m}$ and forward propagation

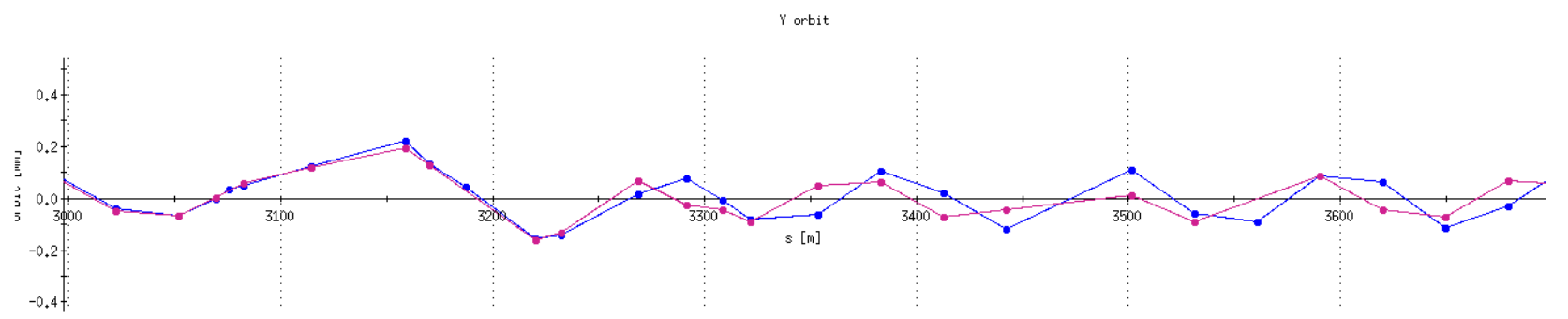

FIG. 10. Expaned view of Fig. 9 showing that the deviation originating from IR4

Then, a different range (from 3400 to $3800 \mathrm{~m}$ ) was used for the fitting, and the fitted orbit was backward propagated. The result from forward propagation was confirmed by the backward propagation since the orbits start to deviate in the same region.

In this example the orbit fit program confirmed that the source of diurnal variation of RHIC orbit is in IR4 region, which is suspected to be the cryogenic pipe, however, further narrow down of the longitudinal position of the source is needed. The precision of localizing error sources by orbit fit program is limited by the spacing of BPMs, which is tens of meters at RHIC. 


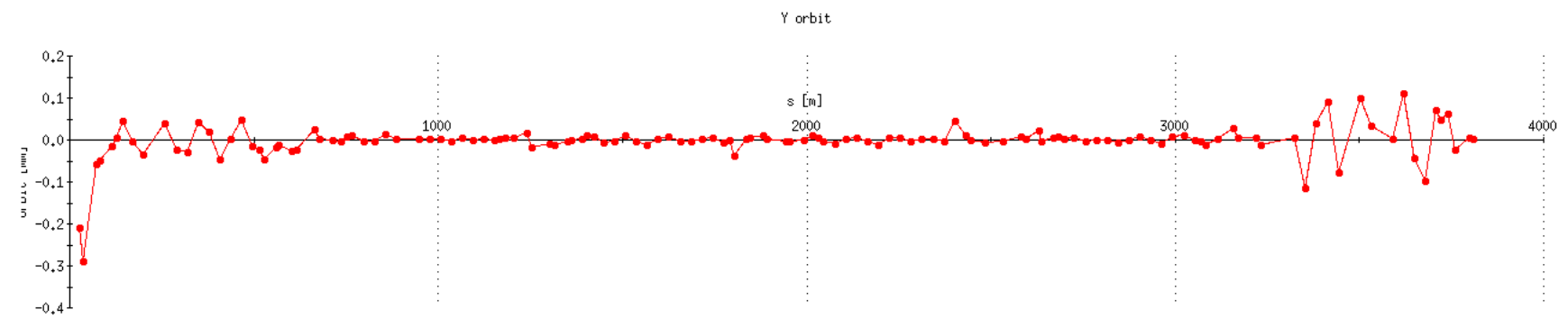

FIG. 11. The difference of original difference orbit and forward propagated fit orbit

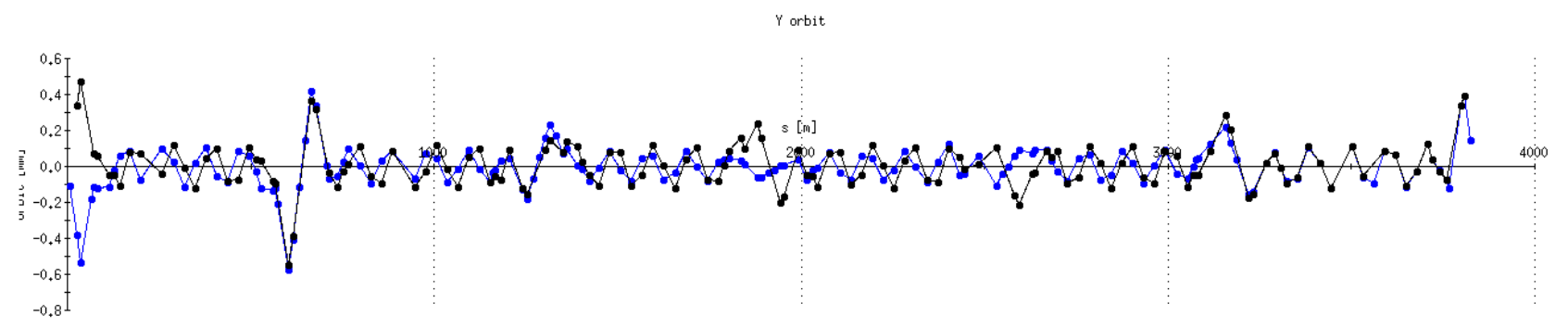

FIG. 12. The vertical difference orbit (blue) vs fitted orbit (black) using range from 3400 to $3800 \mathrm{~m}$ and backward propagation

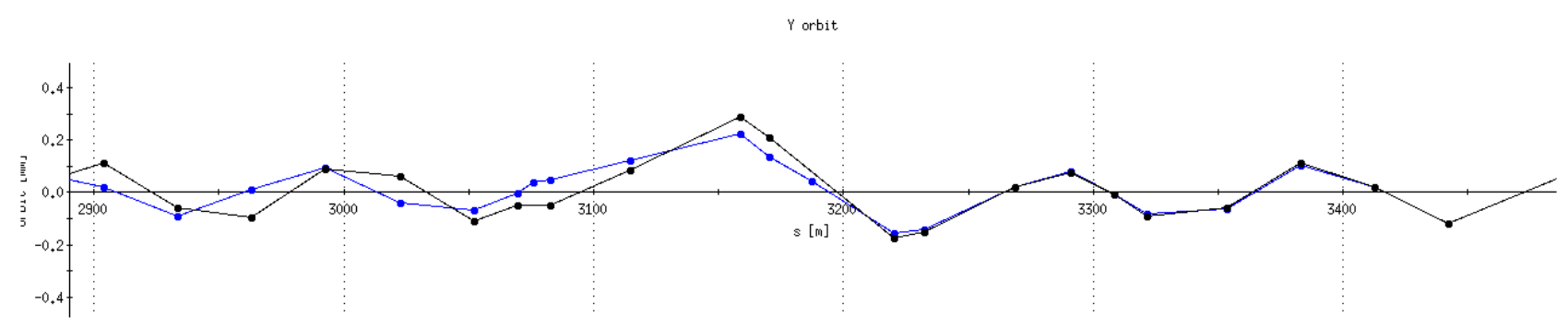

FIG. 13. Expaned view of Fig. 12 showing that the deviation originating from IR4

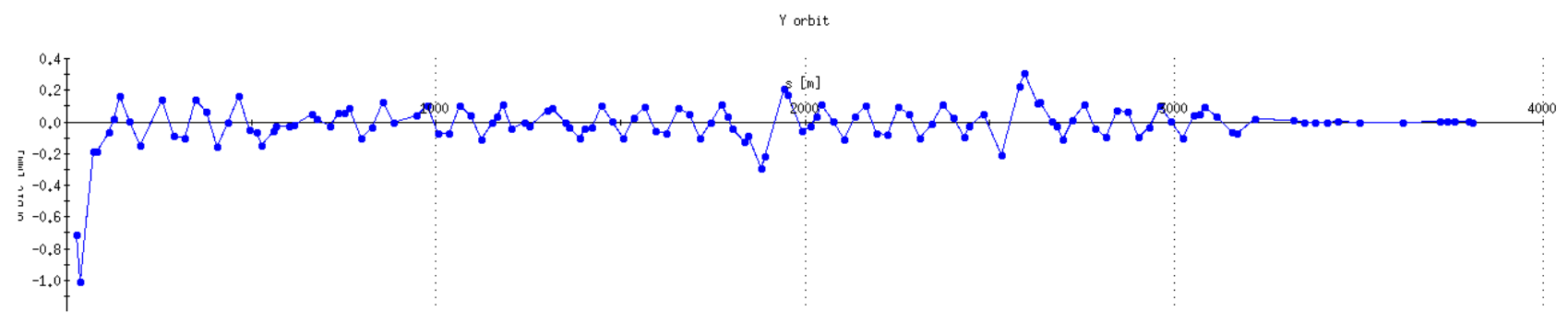

FIG. 14. The difference of original difference orbit and backward propagated fit orbit

\section{POSSIBLE FUTURE APPLICATION}

In principle the algorithm given here could be applied on a much faster time scale using as input data from the BPMs acquired turn-by-turn.

As a case in point, some errors in RHIC could degrade the beam emittance seriously and take hours, sometimes days to be found or never being found. The blue abort kicker and yellow spontaneous debunching are the two most notorious problems in Run-11, of which the first one was eventually found by turning off element by element, the latter one disappeared before the source could be found. With the new orbit fit function, the hunting for error sources could be much easier.

What makes the search for the error source difficult for the two mentioned cases is, the sources are intermittent 

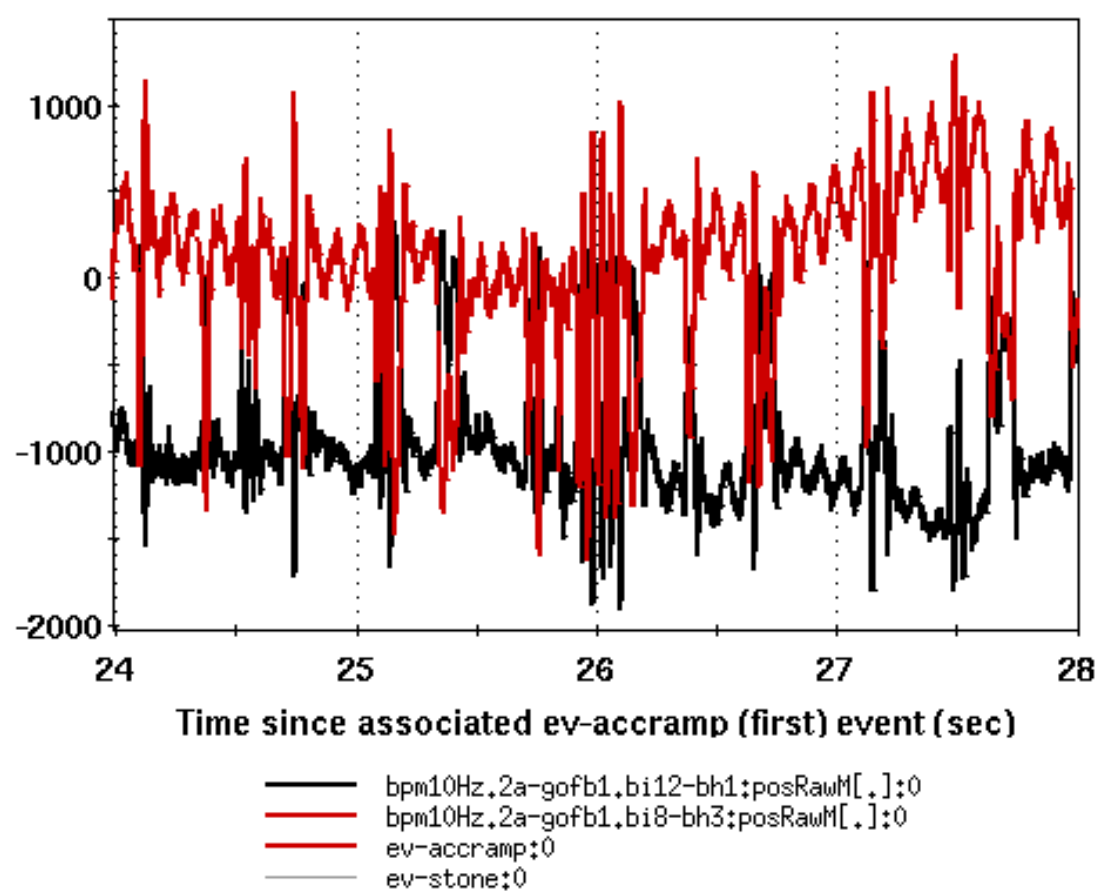

FIG. 15. Measurements from two fast BPMs (bi12-bh1, bi8-bh3) during the bunch lengthening problem

instead of static. This is clear from the fast BPM measurement (Fig. 15), taken while the bunch lengthing was present.

Fortunately, the turn-by-turn BPMs can be configured to take measurements with spacing other than 1 turn. By varying the spacing of two consecutive measurements, one would be able to record orbits with and without the error source kicking the beam. Then, one could locate the error source by following the demonstrated procedure.

\section{CONCLUSION}

An orbit fit algorithm for both transport lines and storage rings was presented. The algorithm has been implemented into RhicOrbitDisplay. Offline tests using BPM polarity check data validated its potential for hunting error sources in RHIC. Its application supported previous analysis suggesting that the motion of quadrupole magnets near the cryogenic feed pipe in IR4 are responsible for the diurnal drift seen in RHIC. A short user's guide is given in Appendix.

\section{ACKNOWLEDGMENTS}

C. Liu would like to thank J. Kewisch and Ted D' Ottavio for the help on programming, R. Michnoff on turn-by-turn BPM configuration and M. Blaskiewicz for discussions.

We would also like to acknowledge P. Emma (SLAC)'s initial application at the SLC.

We thank also A. Marusic for raising the question of possible "moving magnets" and for clarifying the actions taken years ago (decoupling of the cryogenic lines from the roof versus full and complete decoupling).

\section{Appendix: User's guide}

In RhicOrbitDisplay, clicks orbit in the menu bar. A pull down menu appears which has the new Fit Orbit at the bottom (Fig. 16).

Clicking on Fit Orbit leads to the Orbit Fit window (Fig. 17), which resembles the Orbit Correction window. 


\begin{tabular}{l}
\hline Orbit Correction \\
\hline Rescale Plot \\
\hline Clear All Orbits \\
Plot Bad Data \\
F Plot DX and spin rotator BPMs \\
r Auto-Unselect \\
Plot: \\
\hline Subtract baseline \\
Take orbit as baseline \\
Display baseline \\
\hline Create Difference Orbit \\
Create Averaged Orbit \\
Calculate Dispersion \\
Fit Orbit
\end{tabular}

FIG. 16. RhicOrbitDisplay pull down menu

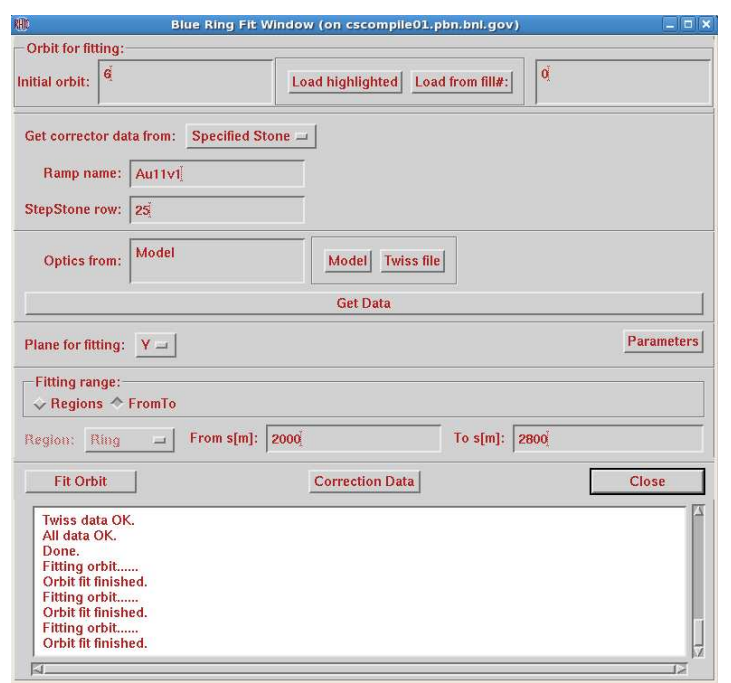

FIG. 17. Orbit fit window

[1] M. Minty and F. Zimmermann, Measurement and control of charged particle beams (Springer Verlag, 2003).

[2] Y. Cai, M. Donald, J. Irwin, and Y. Yan, in Particle Accelerator Conference, 1997. Proceedings of the 199'7, Vol. 2 (IEEE, 1997) pp. 2583-2585.

[3] V. Ptitsyn, private communications.

[4] V. Ptitsyn, A. Marusic, R. Michnoff, M. Minty, G. Robert-Demolaize, and T. Satogata, in Proceedings of Beam Instrumentation Workshop (2010)

[5] D. Trbojevic, private communications. 